\title{
What roles do tonic inhibition and disinhibition play in the control of motor programs?
}

\author{
Paul R. Benjamin*, Kevin Staras and György Kemenes \\ Sussex Centre for Neuroscience, School of Life Sciences, University of Sussex, Brighton, East Sussex, UK
}

\section{Edited by:}

William Kristan,

UC San Diego, USA

Reviewed by:

Rhanor Gillette,

University of Illinois at

Urbana-Champaign, USA

Klaudiusz Weiss,

Mount Sinai School of Medicine, USA

*Correspondence:

Paul R. Benjamin, School of Life

Sciences, University of Sussex,

Brighton BN1 9QG, UK

e-mail:p.r.benjamin@sussex.ac.uk
Animals show periods of quiescence interspersed with periods of motor activity. In a number of invertebrate and vertebrate systems, quiescence is achieved by active suppression of motor behavior is due to tonic inhibition induced by sensory input or changes in internal state. Removal of this inhibition (disinhibition) has the converse effect tending to increase the level of motor activity. We show that tonic inhibition and disinhibition can have a variety of roles. It can simply switch off specific unwanted motor behaviors, or modulate the occurrence of a motor response, a type of 'threshold' controlling function, or be involved in the selection of a particular motor program by inhibiting 'competing' motor mechanisms that would otherwise interfere with the carrying out of a desired movement. A suggested general function for tonic inhibition is to prevent unnecessary non-goal directed motor activity that would be energetically expensive. The reason why basic motor programs might be a particular target for tonic inhibition is that many of them involve central pattern generator circuits that are often spontaneously active and need to be actively suppressed for energy saving. Based on this hypothesis, tonic inhibition represents the default state for energy saving and motor programs are switched-on when required by removal of this inhibition.

Keywords: tonic inhibition, disinhibition, motor program selection, modulation, behavioral switching

\section{BACKGROUND}

There are many examples in the neuroethological literature where animals show periods of reduced motor activity or quiescence and this makes evolutionary sense because it reduces unnecessary energy expenditure and potentially makes animals less conspicuous to predators (Lambert et al., 2004a). In a number of systems this reduction of motor activity is due to tonic inhibition of motor circuits induced by sensory inputs or changes in internal state. More recently it has been argued that reduction of the level of this prolonged tonic inhibitory activity ('disinhibition') has the converse effect, that is to increase activity in motor programs (Mink, 1996; Grillner et al., 2005). Thus tonic inhibition has the potential to both suppress and activate motor behavior depending on the level of electrical firing in the inhibitory pathways, acting as a type of neural switching mechanism. Other types of inhibition occur within motor circuits but these have different roles in motor behavior. For instance inhibitory synaptic connections form part of a central pattern generating mechanism for rhythmic movement (reciprocal inhibition, Perkel and Mulloney, 1974) or modify the strength of reflexive responses (recurrent inhibition, Fischer and Carew, 1997). Historically, inhibition and disinhibition were proposed to be involved in the selection of motor programs and ethologists suggested that many behaviors were likely to be under inhibitory control as part of a hierarchical mechanism to allow high priority behaviors to be preferentially selected (Tinbergen, 1951; McCleery, 1983). Disinhibition led to the activation of behaviors that were lower in the hierarchy. At the neuronal level behavioral selection has been shown to involve inhibitory interactions between networks (reviewed by Kristan and Gillette, 2007; Kristan, 2008) but often more complex dynamic processes occur in populations of interneurons and inhibition forms only part of the mechanism for decision-making.
The recent increase in information on the identification of the circuit level sensory and interneuronal mechanisms underlying tonic inhibition and its disinhibition in a number of vertebrate and invertebrate motor circuits has allowed us to ascertain the general features of this type of motor control and to make a case for its general importance in the selection of motor programs across a range of invertebrate and vertebrate systems.

\section{MODULATORY ROLE FOR TONIC INHIBITION}

One of the first and still best examples where tonic inhibition was shown to play an important role in behavioral modulation came from the Krasne Laboratory (Krasne and Wine, 1975; Vu and Krasne, 1993; Vu et al., 1993) working on the crayfish tailflip escape response (Figure 1A). Tonic inhibition reduces the excitability of paired lateral giant fibers (LG), command neurons that mediate the escape behavior, making it less likely that escape behavior will occur. A variety of different circumstances are now known that cause suppression of the escape behavior via tonic inhibition including feeding, restraint that hinders the execution of escape, and agonistic encounters with other crayfish (Krasne and Lee, 1988; Vu et al., 1993; Krasne et al., 1997). The neural organization of these tonic inhibitory effects make the escape behavior highly adaptive and interactive with other behavioral systems. Vu et al. provided an elegant explanatory model for how this works. With no absolute ability to prevent escape responses, they propose that tonic inhibition helps to set a variable threshold for the occurrence of escape behavior by interacting with the various types of excitatory inputs that normally evoke LG activity and escape. In this model an increase in tonic synaptic inhibition due to constraint or feeding sets a high threshold for escape whereas 


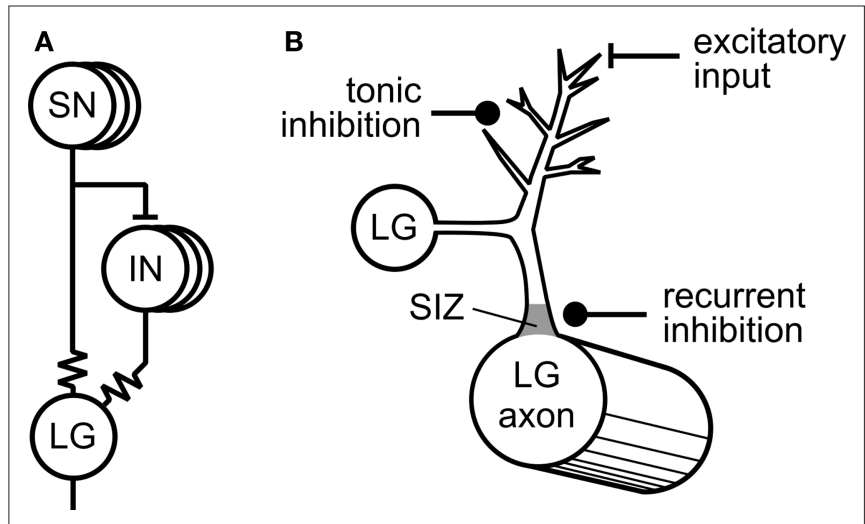

FIGURE 1 |Tonic inhibition of the crayfish escape system (adapted from Edwards et al., 1999 with permission from Elsevier). (A) Mechanosensory neurons (SN) directly excite the Lateral Giant (LG) interneuron via an electrotonic synaptic connection (resistor symbol). The SNs also excite mechanosensory interneurons (INS) that are also electrotonically coupled to the LG. (B) Synaptic inputs to the LG. Inhibitory synapses responsible for tonic inhibition are located distally from the LG cell body as are the excitatory synaptic inputs. Synaptic inputs responsible for recurrent inhibition are located proximally to the LG cell body close to the spike initiating zone (SIZ).

strongly excitatory sensory inputs that result from threatening stimuli lower the threshold for escape. At the cellular level, the tonic inhibitory synapses are located on the distal dendritic processes of the LG neuron at a considerable electrotonic distance from the spike initiation zone near to the LG cell body. The interactions with excitatory synaptic inputs occur at the same distal dendritic sites (Figure 1B). Because of the distal location of the inhibitory synapses, tonic inhibition can never completely prevent the effects of competing excitatory inputs that if sufficiently strong can override the effects of tonic inhibition (by disinhibition). Vu et al. (1993) contrasted this type of distal inhibitory process with another type of synaptic inhibition, recurrent inhibition, also acting on LG. The proposed role of this second type of inhibition of the LG is to prevent a further sensory activation of the escape response during an already-triggered escape sequence. Unlike tonic inhibition, the synapses mediating recurrent inhibition lie close to the spike-initiating zone in LG (Figure 1B) and always rapidly prevent LG spiking in an absolute way despite the presence of conjoint excitatory sensory inputs.

The long-term modulatory effects of tonic inhibition are striking. In naïve animals, there is a gradual build-up in the strength of tonic inhibition as measured by the threshold for LG firing when restraint is applied to an intact preparation ( $\mathrm{Vu}$ et al., 1993). After a few minutes of restraint tonic inhibition begins to be effective but it takes about $40 \mathrm{~min}$ to completely inhibit escape and then it continues to have inhibitory effects on LG firing for several hours after removal of the restraint. The mechanism for this sustained activity in the tonic inhibitory pathway is unknown but does not appear to be due to activation of second messenger pathways that cause persistent effects in other systems. Tonic inhibition in crayfish, like recurrent inhibition, is mediated by GABAergic classical type A receptors that mediate short-lasting effects probably on chloride channels (Vu and Krasne, 1993).
The modulatory role of tonic inhibition on behavior is emphasized also by work on the feeding system of the snail Lymnaea stagnalis (Figure 2) from our own laboratory (Staras et al., 2003) but here the level of modulation by tonic inhibition depends on internal state, hunger, and satiety (see Figure 2C).

Feeding in Lymnaea consists of sequences of rhythmic biting (leading to food ingestion) interspersed with periods of quiescence due to tonic inhibition. Tonic inhibition is provided by one of the CPG interneurons, the N3t cell. During quiescence this cell fires continuously and prevents activity in the rest of the CPG network via a monosynaptic inhibitory connection with another (CPG) interneuron, the N1M. However, in the presence of food, the N3t cell is hyperpolarized reducing the level of tonic inhibition to the $\mathrm{N} 1 \mathrm{M}$ and this has a permissive role in allowing the feeding CPG to oscillate (Figure 2D). Under these conditions the N3t becomes part of the CPG firing in a rhythmic pattern and provides phasic feedback to the N1 rather than continuous inhibition (Figure 2E). The absolute level of suppressive control is determined by the animal's behavioral state with the level of inhibition (firing rate of $\mathrm{N} 3 \mathrm{t}$ ) reduced by hunger and increased by satiety (Figure $2 \mathrm{C}$ ). Like the crayfish, tonic inhibition of feeding in the snail can be modeled as part of a variable threshold system. Satiety acts to raise the threshold for feeding whereas hunger reduces the threshold. By reducing the firing rates of the $\mathrm{N} 3 \mathrm{t}$ cell, food disinhibits the feeding CPG resulting in feeding behavior. A recent computational study (Vavoulis et al., 2007) was able to simulate the N3t-mediated effects of hunger and satiety on the feeding pattern. Varying the frequency of N3t tonic synaptic inputs in accordance with those occurring in hungry and satiated animals, the model was able to reproduce the patterns of feeding activity seen in the biological system providing further evidence for the role of tonic inhibition in behavioral state regulation of feeding.

Modulation of tonic inhibition originating from within a rhythm generating circuit is not confined to this example from an invertebrate but has also been reported to occur within the swallow circuit of rats (Wang and Bieger, 1991). The medullary pattern generator for swallowing maintains an inactive state as a result of endogenous GABAergic tonic inhibition acting on solitarial premotor elements of the swallowing CPG. This tonic inhibition counteracts impinging excitatory inputs acting on premotor neurons via NMDA and muscarinic receptors. The mechanism of disinhibition of the GABAergic tonic inhibition that would facilitate pharyngeal and esophageal co-ordinated swallowing is unclear.

\section{SENSORY CONTROL OF TONIC INHIBITION}

This has been best studied in the hatching Xenopus tadpole (Roberts et al., 2008). In no other system have the sensory processes leading to tonic inhibition at the cellular level been so well investigated. Newly hatched tadpoles spend most of their time hanging immobile from a strand of mucus secreted from the cement gland on the head. Swimming is largely suppressed over several days and only occurs for short periods when the tadpoles are temporarily detached from their substrate. Sensory stimuli that normally induce swimming bouts in older tadpoles, like light dimming, are ineffective in triggering swim behavior in newly hatched tadpoles in these first few days of life (Lambert et al., 2004a). Suppression of swim activity is due to tonic inhibition by a class of hind brain reticulo-spinal (mid hind brain 

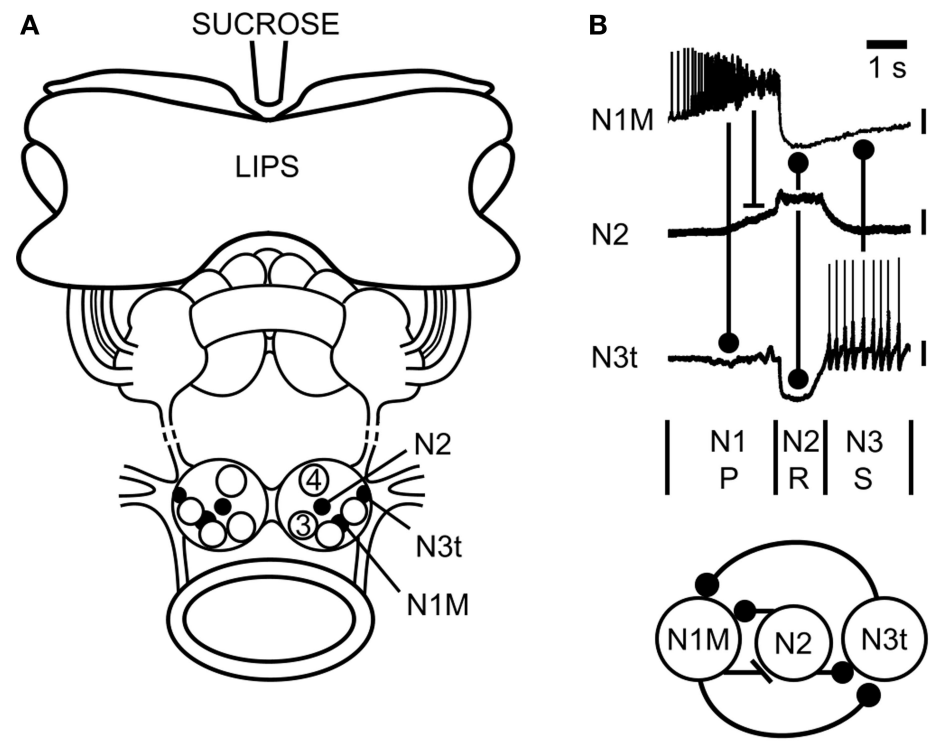

C

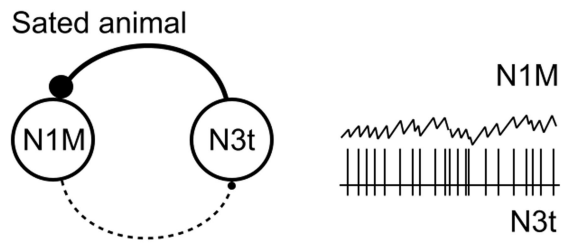

Hungry animal
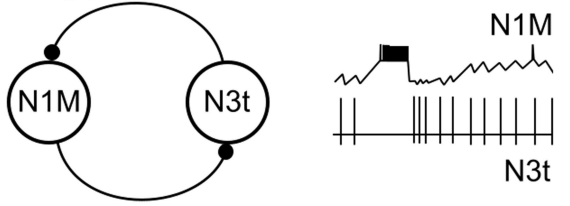

Feeding animal
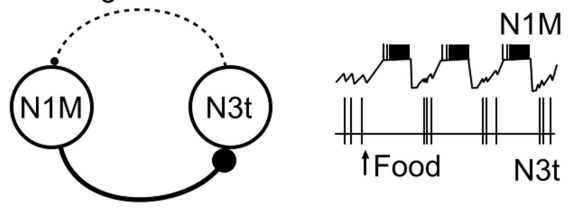

D

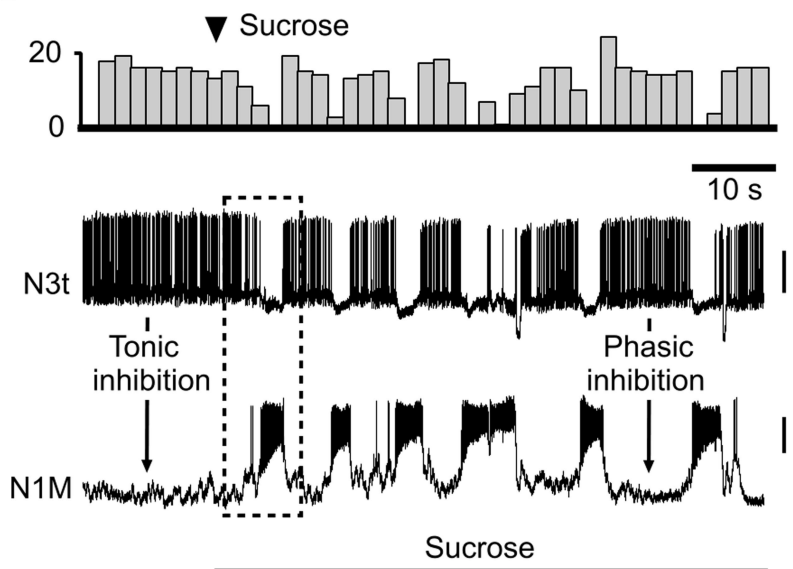

E

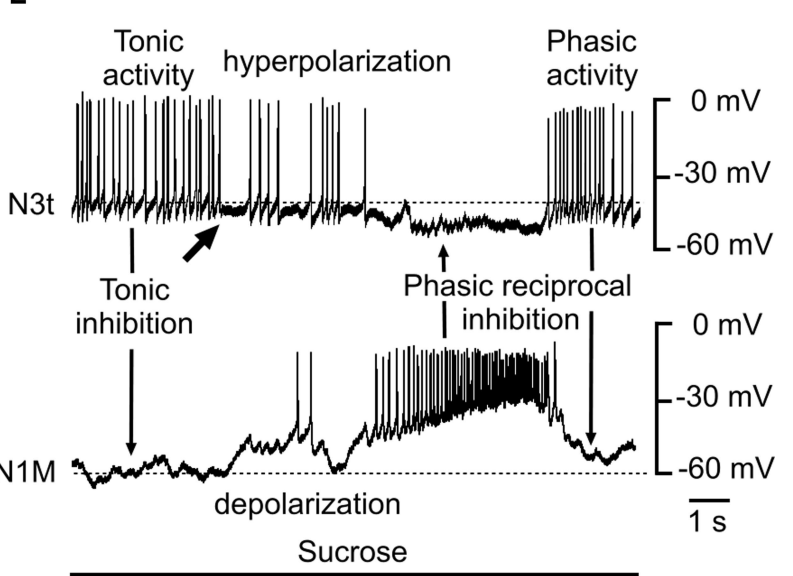

FIGURE 2 | Modulation of the snail feeding system by tonic inhibition (adapted from Staras et al., 2003, with permission from Elsevier). (A) The semi-intact preparation used for electrophysiological recording showing the location of feeding CPG interneurons in the buccal ganglia. A feeding stimulus, sucrose, is applied to the lips. (B) Firing patterns (top) and synaptic connections (bottom) of the three main types of interneurons that form the feeding central pattern generator. One cycle of feeding activity is shown with the origins of the synaptic inputs indicated by connecting bars. The N1M fires during the first protraction (P) phase, the N2 (with truncated spikes) the second rasp (R) phase and the N3t the third swallow (S) phase of the feeding cycle. (C) Model summarizing the modulatory effects of satiation and hunger on the tonic inhibition of the feeding pattern. In satiated animals the N3t fires continuously and the consequent inhibitory effects on the N1M prevent bursting in this cell. In hungry animals, even with no food present, there are occasional feeding bursts in the N1M due to the lower rate of firing of the N3t. In feeding animals the tonic N3t is weak and insufficient to prevent sustained bursting in the N1M and the N3t fires phasically to become part of the feeding

reticulospinal, MHR) neurons (Lambert et al., 2004b) whose axons provide descending GABAergic inhibitory synaptic inputs to the spinal centers generating rhythmic swimming movements (Figure 3A). A second type of hind brain neuron, the rostral hind brain (RH) type, has no spinal projections but has indirect effects on hind-brain

rhythm. The thickness of the continuous lines connecting the N1M and N3t cells (left) indicates the strength of the inhibitory effects between the cells and the dashed lines the absence of phasic inhibitory effects. (D) An experiment in the semi-intact preparation showing that a food stimulus reduces the suppressive inhibitory control by the N3t cell and releases rhythmic fictive feeding activity. The change in the pattern of N3t firing is emphasized by the top trace where the number of N3t spikes is accumulated in 3s bins. (E) Expansion of the boxed area shown in (D) shows the first cycle of fictive feeding activity in the N1M after the sucrose-induced reduction in N3t firing rate. The arrow under the N3t trace indicates the point at which N3t starts to hyperpolarize and its tonic spike frequency begins to decrease. This decrease in N3t firing is followed by complete suppression of N3t firing when the N1M becomes active and synaptically inhibits the N3t. The subsequent phasic N1M-N3t reciprocal inhibition leads to the alternating patterns of N1M/N3t firing seen throughout the feeding pattern that follows. In (A) to (C) a stick indicates an excitatory synaptic connection between neurons and a ball indicates an inhibitory synaptic connection.

outputs to the spinal cord by providing excitatory synaptic inputs to the MHRs. Both the MHRs and the RHs receive direct excitatory sensory inputs from trigeminal mechanosensory neurons innervating the cement gland (Figure 3A). Experiments where it was possible to mimic the natural mechanical stimulation to the cement gland 
A Tadpole swim suppression

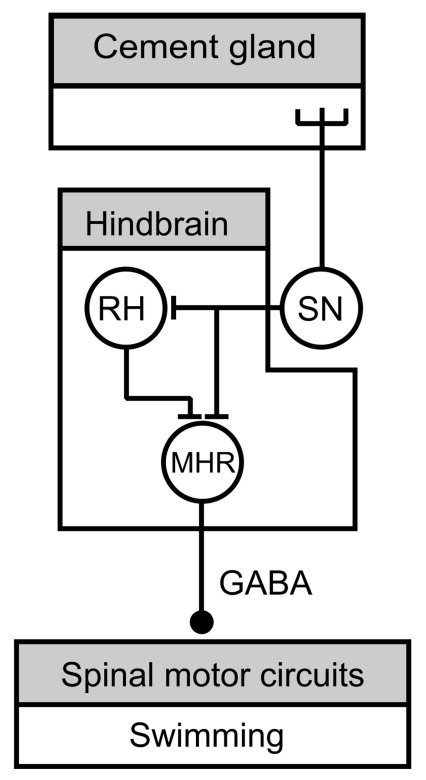

FIGURE 3 | Summary of neural circuits underlying tonic inhibition of tadpole swimming and motor pattern selection by the vertebrate basal ganglia (adapted from Lambert et al., 2004a (A) and Grillner et al., 2005 (B), with permission from Springer and Elsevier, respectively). (A)Tension in the mucus secreted by the head cement gland during attachment of the tadpole to a substrate like the edge of a dish, activates cement gland mechanosensory neurons (SN). The sensory neurons directly excite rostral hind brain $(\mathrm{RH})$ neurons and mid hind brain reticulospinal neurons (MHR). The $\mathrm{RH}$ neurons excite the MHRs and provide an indirect route for effects on spinal cord circuitry. The MHR neurons provide GABAergic inhibition of the spinal swim circuits. During the more-or-less continuous attachment of the tadpole during the first post-hatch day sustained tonic activity in the cement gland sensory neuron leads to corresponding tonic activity in the MHR followed by suppression of swim behavior. (B) Cortical and thalamic areas provide
B Basal ganglia behavioral selection

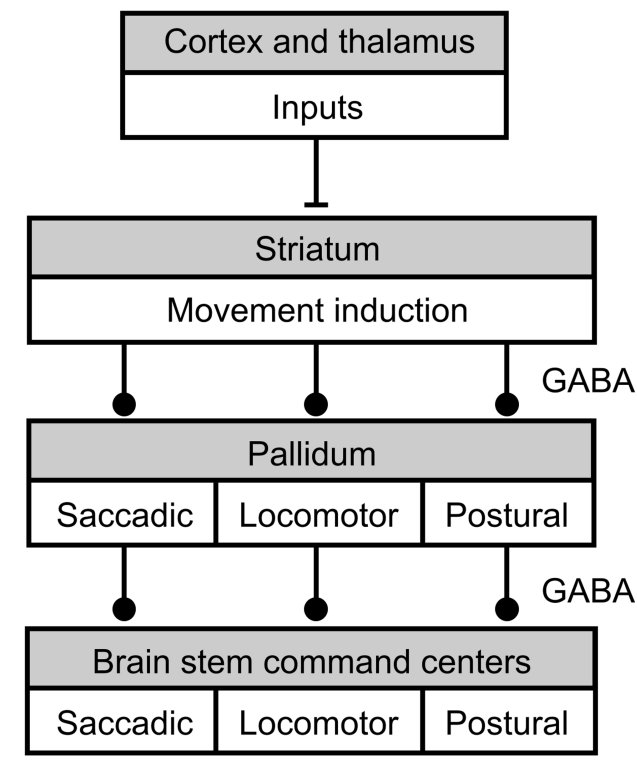

excitatory synaptic connections to the striatal input area of the basal ganglia that is involved in movement induction and selection. Striatal neurons activate brain stem command centers by inhibiting the firing of pallidal neurons. In the absence of striatal activity, pallidal neurons fire at high rates and provide tonic inhibition to brain stem command centers that are responsible for the activation of motor programs for saccadic eye movements, locomotion and posture. The striatum thus provides a disinhibitory mechanism for the generation of basic movements. Striatal and pallidal control pathways are functionally organized to allow the selective disinhibition of particular motor programs. This is indicated by the division of motor programs into boxed compartments with a corresponding segregation of the inhibitory connecting pathways. All the inhibitory connections in these circuits are GABAergic. A stick indicates an excitatory synaptic connection between neurons or nuclei, and a ball an inhibitory synaptic connection. by applying weights to the mucus in inverted tadpoles showed that individual trigeminal mechanosensory afferents fired continuously for as long as the stimulus was maintained (up to one hour, Lambert et al., 2004a). It therefore seems likely that these sensory neurons fire throughout the period of swimming suppression and are responsible for the inhibition of the down-stream spinal circuits. The MHRs show similar long-term increases in tonic spiking due to mechanical stimulation and the excitatory synaptic input from the $\sim 40$ sensory neurons appears to be capable of driving MHR spike activity (modeled in Lambert et al., 2004b). The average firing rate of the caudal MHRs in the attached state is low, $\sim 0.5 \mathrm{~Hz}$ so it was important to show that that was sufficient to prevent firing in the tadpole swimming motoneurons. The duration of IPSPs in motoneurons due to MHR inhibition are known (120-230 ms) and simulations reveal that IPSP with durations of $130 \mathrm{~ms}$ are effective in inhibiting motoneurons for $70 \%$ of the time. This compares well with the lack of responsiveness of tadpoles to dimming in behavioral experiments during attachment which is also $\sim 70 \%$ (Lambert et al., 2004b).

The relative simplicity of the Xenopus tadpole circuitry compared with mammals has allowed the production of a highly detailed and convincing analysis of the role of tonic inhibition in the early post-hatching stages of the tadpole's life. Whether it plays a similar role in periods of quiescence that occur in the later stages of the tadpole development when the tadpoles show greater levels of swimming activity and other behaviors such as feeding has not been investigated. It is interesting that the same circuit that underlies sustained reduction in swim activity is also involved in more phasic 'stopping' of swimming (e.g., Perrins et al., 2002) that occurs when a tadpole bumps into solid objects. Under these circumstances pressure on the cement gland or other parts of the head skin stops on-going swimming activity by brief activation of trigeminal afferents and the reticulo-spinal neurons. This result emphasizes the importance of maintained sensory input in determining the duration of inactivity. In the crayfish escape system tonic inhibition due to restraining stimuli could outlast the period of external sensory stimulation (Vu and Krasne, 1993) suggesting the presence of additional sustaining mechanisms.

\section{SELECTION OF MOTOR PROGRAMS IN VERTEBRATES BY TONIC INHIBITION AND DISINHIBITION}

As well as the control of individual motor behaviors evidence is accumulating that tonic inhibition and disinhibition are involved in the selection of particular motor programs from the many that 
underlie basic motor behaviors in vertebrates like locomotion, postural tone, breathing, eye movements, and feeding. A general interpretation of the evidence suggests that unwanted motor programs are inhibited by tonic inhibition and required ones are recruited by selective disinhibition (removal of tonic inhibition). An increasing amount of detailed evidence from a variety of vertebrates, where data from homologous structures can be compared, suggest that this method of selection of motor programs is carried out by the collection of motor nuclei known as the basal ganglia (Chevalier and Deniau, 1990; Mink, 1996; Grillner et al., 2005). Although the circuitry involving the various diverse functions of the basal ganglia in motor control is complex and allows interactions with many regions of the brain (e.g., Gerfen, 1992) the circuit that underlie basal ganglia selection of basic 'non-cognitive' motor programs is relatively simple (Figure 3B).

Tonic inhibition of motor programs is mediated by a number of nuclei collectively known as the pallidum that form the output pathways of the basal ganglia (Figure 3B). Pallidal neurons fire continuously at high resting rates $(50-100 \mathrm{~Hz})$ and provide tonic inhibition to brain stem and tectal structures that act as command and control centers for movement and posture (Hikosaka et al., 2000, eye movements; Orlovsky et al., 1999, locomotion; Takakusaki et al., 2004, postural tone). Under these default conditions of high pallidal spike activity motor behaviors are generally suppressed. How this pallidal activity suppresses movement at the network level is best understood for locomotion and the circuits involved are conserved in all groups of vertebrates from mammals to lampreys (Grillner, 2003; Ménard et al., 2007; Ménard and Grillner, 2008). Locomotory rhythmic activity generated by spinal CPGs is initiated and maintained by repetitive electrical stimulation of diencephalic or mesencephalic locomotory regions (DLR, MLR) (Takakusaki, 2008) and based on a variety of electrophysiological and pharmacological evidence these centers are considered to be command centers for locomotion (Grillner, 2003; Grillner et al., 2005). GABAergic nerve fibers that mainly originate from the globus pallidus interna region of the pallidum directly innervate these locomotory command regions (Chevalier and Deniau, 1990) and pharmacological evidence (e.g., Ménard et al., 2007; Ménard and Grillner, 2008) supports the conclusion that these GABAergic pallidal fibers underlie the tonic inhibition of the MLR and DLR by the pallidum. In the absence of tonic inhibition from the pallidum, the DLR, and MLR outputs monosynaptically excite descending reticulo-spinal interneurons that in turn activate spinal CPG and motoneuronal networks. Pharmacological experiments show that rhythmic motor activity in spinal neurons can be released from pallidal tonic inhibition by injecting a $\mathrm{GABA}_{\mathrm{A}}$ antagonist into the locomotory command centers (e.g., Ménard and Grillner, 2008).

The striatal input region of the basal ganglia (Figure 3B) plays a key role in the activation of motor programs by suppressing tonic activity in the pallidal output neurons. In contrast to the pallidum neurons, striatal neurons (the predominant group are the medial spiny output neurons) have a high threshold for activation and are normally silent due to a high resting potential unless they receive excitatory input from the cerebral cortex, thalamus, or limbichypothalamic regions (Gerfen, 1992; Mink, 1996; Takakusaki, 2008). These excitatory synaptic inputs alone are ineffective in activating the spiny output neurons unless dopamine acting on
D1 receptors is present to increase their excitability (reviewed in Thompson et al., 2008). One important population of spiny output neurons are GABAergic and these neurons have direct inhibitory effects on pallidal output neurons. These inhibitory effects on pallidal neurons are strong enough to prevent or reduce tonic firing so that the motor command centers are released from tonic inhibition with the consequent activation of motor programs. A variety of anatomical and electrophysiological experiments suggest that the striatal-pallidal input and output pathways are organized to allow for the selective disinhibition of particular motor programs (see Figure 3B). Data indicate that the control system is functionally divided so that sub-populations of striatal neurons provide inhibition to a subpopulation of pallidal neurons that in turn control the activity of a particular motor program. Evidence for this type of organization comes from experiments where electrical or pharmacological stimulation is applied locally to a particular subgroup of striatal neurons (reviewed in Grillner et al., 2005). This results in the activation of a particular motor program such as those underlying locomotion or saccadic eye movements.

Disinhibition can be seen as a key process in the expression of striatal functions and functional division of this process within the basal ganglia the main mechanism by which different basic motor programs are selected. As well as divisions of control functions within the basal ganglia, the topographical organization of extrinsic inputs to the basal ganglia (e.g., from the cerebral cortex) also probably play a role in the selection of motor programs, although the details of how this is organized are unknown. A final point is that there are other neural pathways in the vertebrate brain that are directly involve in movement control without an obvious requirement for the basal ganglia to be part of the decision-making process, for instance the corticospinal pathways that are involved in volitional control of arm and hand movements (e.g., Graziano et al., 2002). Despite this, animals without a cortex (e.g., cats, Bjursten et al., 1976) surprisingly are still able to locomote and carry out basic motor programs like feeding under sensory guidance. This is due presumably to the subcortical inhibitory and disinhibitory mechanisms selection mechanisms we describe here together with other retained mechanisms that include thalamic (sensory) input to the basic ganglia (Grillner et al., 2005).

\section{CONCLUSIONS AND DISCUSSION}

Tonic inhibition and disinhibition has a variety of roles varying from modulation of motor programs, a type of a 'threshold' controlling function (crayfish escape, snail feeding), to switching off an individual motor programs (tadpole swim) to the selection of alternative motor programs (basal ganglia). In the latter example, the basal ganglion has been viewed as an action selection device (Gurney et al., 1998) that inhibits 'competing' motor mechanisms that would otherwise interfere with the carrying out of desired movements. On this model tonic inhibition acts as brake on the target systems that might need to access the same motor effector organs (muscle groups/limbs). An obvious general function for tonic inhibition is to prevent unnecessary non-goal directed activity that would be energetically expensive. The reason why motor programs might be a particular target for tonic inhibition is that many of them involve central pattern generator circuits (locomotion, feeding) that are often 'spontaneously' active and need to 
be actively suppressed for energy saving. On this argument tonic inhibition represents a default state for energy saving and activity can be switched on by removal of this inhibition. The ability to rapidly switch on behavior by disinhibition is favored by tonic inhibition being mediated by 'fast' $\mathrm{GABA}_{\mathrm{A}}$-ergic receptors, whose effects on target neurons can rapidly reversed by cessation of tonic firing. It should be noted that in many examples of behavioral activation,

\section{REFERENCES}

Bjursten, L.-M., Norsell, K., and Norsell, U. (1976). Behavioural repertoire of cats without cerebral cortex from infancy. Exp. Brain Res. 25, 115-130.

Chevalier, G., and Deniau, J. M. (1990). Disinhibition as a basic process in the expression of striatal functions. Trends Neurosci. 13, 277-280.

Edwards, D. H., Heitler, W. J., and Krasne, F. B. (1999). Fifty years of a command neuron: the neurobiology of escape behavior in the crayfish. Trends Neurosci. 22, 153-161.

Fischer, T. M., and Carew, T. J. (1997). Activity-dependent regulation of neural networks: the role of inhibitory synaptic plasticity in adaptive gain control in the siphon withdrawal reflex of Aplysia. Biol. Bull. 192, 164-166.

Gerfen, C. R. (1992). The neostriatal mosaic: multiple levels of compartmental organization. Trends Neurosci. $15,133-139$

Graziano, M. S. A., Taylor, C. S.R., Moore, T., and Cooke, D. F. (2002). The cortical control of movement revisited. Neuron 36, 349-362.

Grillner, S. (2003). The motor infrastructure: from ion channels to neuronal networks. Nat. Rev. Neurosci. 4 , 573-586.

Grillner, S., Hellgren, J., Menard, A., Saitoh, K., and Wilkstrom, M. A. (2005). Mechanisms for selection of basic motor programs-roles for the striatum and pallidum. Trends Neurosci. 28, 364-370.

Gurney, K. N., Prescott, T. J., and Redgrave, P. (1998). "The basal ganglia viewed as an action selection device," in Proceedings of 8th Annual Conference on Artificial Neural Networks Skovde, Sweden, 1033-1038.

Hikosaka, O., Takaikawa, Y., and Kawagoe, R. (2000). Role of basal ganglia in the control of purposive saccadic eye movements. Physiol. Rev. 80, 9530-9978.

Krasne, F. B., and Lee, S. C. (1988). Response-dedicated trigger neurons as control points for behavioural actions: selective inhibition of lateral giant command neurons during feeding in crayfish. J. Neurosci. 8, 3703-3712.

Krasne, F. B., Shamsian, A., and Kulkrna, R. (1997). Altered excitability of the crayfish lateral giant escape reflex during agonistic encounters. J. Neurosci. $17,709-716$.

Krasne, F. B., and Wine, J. M. (1975). Extrinsic modulation of crayfish escape behaviour. J. Exp. Biol. 63, 433-450.

Kristan, W. B. (2008). Neuronal decision-making circuits. Curr. Biol. 18, R928-R932.

Kristan, W. B., and Gillette, R. (2007). "Decision-making in small neuronal networks," in Invertebrate Neurobiology, eds G. North and R. Greenspan (New York: Cold Spring Harbor Press), 533-554.

Lambert, T. D., Howard, J., Plant, A., Soffe, S., and Roberts,A. (2004a).Mechanisms and significance of reduced activity and responsiveness in resting frog tadpoles. J. Exp. Biol. 207, 1113-1125.

Lambert, T. D., Li, W.-C., Soffe, S. R., and Roberts, A. (2004b). Brainstem control of activity and responsiveness in resting tadpoles; tonic inhibition. J. Comp. Physiol. A 190, 331-342.

McCleery, R. H. (1983). "Interactions between activities," in Animal Behaviour, Vol. 1 Causes and Effects, eds, T. R. Halliday and P. J. B. Slater (Oxford: Blackwell Scientific Publications), 134-167.

Ménard, A., Auclair, F., Bourcier, C., Grillner, S., and Dubuc, R. (2007). Descending GABAergic projections to the mesencephalic locomotor in

excitatory mechanisms are required either to overcome the effects of tonic inhibition (crayfish) or to act as the primary mechanism for motor responses (corticospinal effects on spinal interneurons and motoneurons, Graziano et al., 2002). Indeed for voluntary movements involving the motor cortex, the basal ganglion inhibitory mechanism might best be considered as a gating mechanism for movement control (Chevalier and Deniau, 1990).

the lamprey. J. Comp. Neurol. 501, 260-273.

Ménard, A., and Grillner, S. (2008). Diencephalic locomotor region in the lamprey-afferent and efferent control. J. Neurophysiol. 100, 1343-1353.

Mink, J. W. (1996). The basal ganglia: focused selection and inhibition of competing motor programs. Prog. Neurobiol. 50, 381-425.

Orlovsky, G. N., Deliagina, T. G., and Grillner, S. (1999). Neuronal Control of Locomotion. From Mollusc to Man. New York, Oxford University Press.

Perkel, D. H., and Mulloney, B. M. (1974) Motor pattern production in reciprocally inhibitory neurons exhibiting postinhibitory rebound. Science 185 , 181-183.

Perrins, R, Walford, A., and Roberts, A. (2002). Sensory activation and role of inhibitory reticulospinal neurons that stop swimming in hatchling frog tadpoles. J. Neurosci. 22, 4229-4240.

Roberts, A., Li, W.-C., and Soffe, S. R. (2008). Roles for inhibition: studies on networks controlling swimming in young frog tadpoles. J. Comp. Physiol. A 194, 185-193.

Staras, K., Kemenes, I., Benjamin. P. R., and Kemenes, G. (2003). Loss of selfinhibition is a cellular mechanism for episodic rhythmic behavior. Curr. Biol. $13,116-124$.

Takakusaki, K. (2008). Forebrain control of locomotor behaviors. Brain Res. Rev. 57, 192-198.

Takakusaki, K., Saitoh, K., Harada, H., and Kashiwayanagi, M. (2004). Role of basal ganglia-bain stem pathways in the control of motor behaviors. Neurosci. Res. 50, 137-151.

Tinbergen, N. (1951). The Study of Instinct. Oxford: Oxford University Press, pp 110-113.

Thompson, R. H., Ménard, A., Pombal, M., and Grillner, S. (2008). Forebrain dopamine depletion impairs motor behavior in lamprey. Eur. J. Neurosci. 27, 1452-1460.

Vavoulis, D. V., Straub, S. A., Kemenes, I., Kemenes, G., Feng J.-F., and Benjamin, P. R. (2007). Dynamic control of a central pattern generator circuit: a computational model of the snail feeding network. Eur. J. Neurosci. 25, 2805-2918.

Vu, E. T., and Krasne, F. B. (1993a). Crayfish tonic inhibition: prolonged modulation of behavioural excitability by classical GABAergic inhibition. J. Neurosci. 13, 4394-4402.

Vu, E. T., Lee, S. C., and Krasne, F. B. (1993b). The mechanisms of tonic inhibition of crayfish escape behavior: distal inhibition and its functional significance. J. Neurosci. 13, 4379-4393.

Wang, Y. T., and Bieger, D. (1991). Role of solitarial GABAergic mechanisms in control of swallowing. Am. J. Physiol. Reg. Integr. and Comp. Physiol. 261, 639-646.

Conflict of Interest Statement: The authors declare that the research was conducted in the absence of any commercial or financial relationships that could be construed as a potential conflict of interest.

Received: 16 March 2010; paper pending published: 15 April 2010; accepted: 15 May 2010; published online: 07 June 2010.

Citation: Benjamin PR, Staras $K$ and Kemenes $G$ (2010) What roles do tonic inhibition and disinhibition play in the control of motor programs? Front. Behav. Neurosci. 4:30. doi: 10.3389/fnbeh.2010.00030

Copyright $\odot 2010$ Benjamin, Staras and Kemenes. This is an open-access article subject to an exclusive license agreement between the authors and the Frontiers Research Foundation, which permits unrestricted use, distribution, and reproduction in any medium, provided the original authors and source are credited. 